\title{
Comparison of waveform-derived corneal stiffness and stress-strain extensometry-derived corneal stiffness using different cross-linking irradiances: an experimental study with air-puff applanation of ex vivo porcine eyes
}

\author{
Robert Herber $^{1}$ (D) $\cdot$ Mathew Francis $^{2} \cdot$ Eberhard Spoerl $^{1} \cdot$ Lutz E. Pillunat $^{1} \cdot$ Frederik Raiskup $^{1} \cdot$ Abhijit Sinha Roy $^{2}$
}

Received: 9 January 2020 /Revised: 2 June 2020 / Accepted: 6 June 2020 / Published online: 17 June 2020

(C) The Author(s) 2020

\begin{abstract}
Purpose To assess corneal stiffening of standard (S-CXL) and accelerated (A-CXL) cross-linking protocols by dynamic corneal response parameters and corneal bending stiffness (Kc[mean/linear]) derived from Corvis (CVS) Scheimpflug-based tonometry. These investigations were validated by corneal tensile stiffness (K[ts]), derived from stress-strain extensometry in ex vivo porcine eyes. Methods Seventy-two fresh-enucleated and de-epithelized porcine eyes were soaked in $0.1 \%$ riboflavin solution including $10 \%$ dextran for $10 \mathrm{~min}$. The eyes were separated into four groups: controls $(n=18), \mathrm{S}-\mathrm{CXL}$ (intensity in $\mathrm{mW} / \mathrm{cm}^{2} *$ time in $\left.\min ; 3 * 30\right)$ $(n=18)$, A-CXL $(9 * 10)(n=18)$, and A-CXL $(18 * 5)(n=18)$, respectively. CXL was performed using CCL Vario. CVS measurements were performed on all eyes. Subsequently, corneal strips were extracted by a double-bladed scalpel and used for stress-strain measurements. K[ts] was calculated from a force-displacement curve. Mean corneal stiffness (Kc[mean]) and constant corneal stiffness (Kc[linear]) were calculated from raw CVS data.

Results In CVS, biomechanical effects of cross-linking were shown to have a significantly decreased deflection amplitude as well as integrated radius, an increased IOP, and SP A1 $(P<0.05) . \mathrm{Kc}[\mathrm{mean}] / \mathrm{Kc}[$ linear $]$ were significantly increased after CXL $(P<0.05)$. In the range from 2 to $6 \%$ strain, $\mathrm{K}[\mathrm{ts}]$ was significantly higher in S-CXL $(3 * 30)$ compared to A-CXL $(9 * 10)$, ACXL $(18 * 5)$, and controls $(P<0.05)$. At $8 \%$ to $10 \%$ strain, all protocols induced a higher stiffness than controls $(P<0.05)$.

Conclusion Several CVS parameters and Kc[mean] as well as Kc[linear] verify corneal stiffening effect after CXL on porcine eyes. S-CXL seems to have a higher tendency of stiffening than A-CXL protocols have, which was demonstrated by Scheimpflug-based tonometry and stress-strain extensometry.
\end{abstract}

Keywords Biomechanics $\cdot$ Cornea $\cdot$ Corvis ST $\cdot$ Dynamic Scheimpflug analyzer $\cdot$ Porcine $\cdot$ Stress-strain measurement $\cdot$ Strip extensometry

Robert Herber, Mathew Francis and Abhijit Sinha Roy contributed equally to this work.

This work was presented in parts as poster presentation at ARVO 2018, Honolulu, USA; Annual Conference of the German Society of Ophthalmology 2018, Bonn, Germany; and CXL Experts Meeting 2018, Zurich, Switzerland.

Robert Herber

robert.herber@uniklinikum-dresden.de

1 Department of Ophthalmology, University Hospital Carl Gustav Carus, TU Dresden, Fetscherstraße 74, 01307 Dresden, Germany

2 Narayana Nethralaya Foundation, Bangalore, India

\section{Introduction}

Corneal cross-linking (CXL) was first described by Spoerl and Seiler in 1998 aiming to increase mechanical stiffness of corneal tissue [1]. Several techniques with photosensitizers (e.g., riboflavin), chemical solutions (e.g., glutaraldehyde), and aldehyde sugars (e.g., glucose) were tested for their suitability to increase stiffness primarily in the case of keratectasia [2]. Following these basic investigations, the first clinical study was published by Wollensak et al., who used the procedure of cross-linking with riboflavin and ultraviolet A (UVA) to halt the progression in keratoconic eyes [3]. Keratoconus is described as an ectatic disorder of the cornea [4], which is based on focal biomechanical weakening [5] followed by 
protrusion of the anterior and posterior curvature, corneal thinning, irregular astigmatism, and loss of visual acuity [6].

The most common treatment, based on early investigations of CXL, is the Dresden protocol (standard CXL, S-CXL) where an irradiance of $3 \mathrm{~mW} / \mathrm{cm}^{2}$ is applied for $30 \mathrm{~min}$ on the treated eye after riboflavin is instilled [3]. This is equal to an energy dose of $5.4 \mathrm{~J} / \mathrm{cm}^{2}$ [3] and offers a safety zone to avoid endothelium or retinal damage if the corneal thickness is more than $400 \mu \mathrm{m}$ [7]. According to the Bunsen-Roscoe Law, the photobiological process is reciprocal and leads to a possible change of irradiance with a simultaneous adjustment of the time [8]. Therefore, several CXL protocols were tested with a shorter duration of irradiance to improve the patient's comfort [8] and were introduced into clinical practice as accelerated CXL (A-CXL). Recently, a study was published that compared the clinical outcome after 12 months, in different protocols using topography and tomography of Scheimpflug imaging. All applied protocols showed improvement and stability concerning maximal keratometry, which is currently the most important parameter to verify the efficacy of CXL in vivo [9]. Additionally, S-CXL was more pronounced in improving corneal surface indices provided by Scheimpflug imaging [9].

The evidence of increased stiffness by CXL has so far been shown ex vivo, under laboratory conditions. Stress-strain measurements can be performed as uniaxial tensile tests using an extensometer and have been applied to measure the stiffness and/or Young's modulus in corneal strips of porcine or human donor tissue $[2,8,10,11]$. Another technique to determine the biomechanical properties is the inflation test, where the shape of the cornea is investigated while increasing the intraocular pressure (IOP) [12]. It could be shown that Young's modulus was increased by factor 1.6 in inflation tests, which is comparable to stress-strain measurements in porcine eyes (factor 1.8) [10, 12]. However, these techniques are not applicable in vivo.

In vivo, attempts were made to derive the biomechanical properties of the cornea through special non-contact tonometry methods. There are two commercial devices available, the ocular response analyzer (ORA; Reichert Ophthalmic Instruments, Depew, NY, USA) and the Corvis ST (CVS; Oculus, Wetzlar, Germany). The ORA is a non-contact tonometer that detects corneal deformation after an induced air puff is applied to the cornea. The main ORA parameters corneal hysteresis $(\mathrm{CH})$ and corneal resistance factor $(\mathrm{CRF})$ show lower values in keratoconic eyes compared to healthy ones [13]. However, the performance of discrimination is low for these parameters in keratoconus with normal corneal thickness [13], mild keratoconus [14], and forme fruste keratoconus [15] compared to normal eyes. Furthermore, there could not be found changes after CXL in $\mathrm{CH}$ and CRF [16-18], as they do not describe material stiffness or in others words elasticity [18]. CH is a parameter that takes into account viscosity and elasticity [18]; thus, changes induced by CXL in elasticity might be masked by changes in viscosity [19]. A Scheimpflug-based tonometer, on the other hand, is able to detect more information during the deformation process of the cornea. The Corvis ST is a Scheimpflug analyzer that captures the deformation process of the cornea with an ultra-high-speed camera after applying an air puff [20,21]. In the meantime, the evaluation of Corvis ST measurements has been upgraded by the development of indices that are able to distinguish between healthy and keratoconic eyes [22, 23]. The main index, called the Corvis Biomechanical Index (CBI), is described as a combination of certain dynamic corneal response parameters (DCR) with high sensitivity and specificity in detecting keratoconus [22]. Recently, our group has shown that CBI has greater ability in differentiating between keratoconus and healthy eyes than $\mathrm{CH}$ or CRF [24]. Further, the combination of topographical and tomographical data with biomechanical parameters (Tomographic and Biomechanical Index, $\mathrm{TBI}$ ) is possible and enables a screening for ectasia before refractive surgery is performed [25]. Additionally, alterations in certain DCR parameters suggesting changes in biomechanical properties after CXL in keratoconic eyes have been shown in preliminary [26] and long-term results [27].

Roy and co-workers have introduced a new analytical model that derives corneal and extra-corneal stiffness from the whole deformation process recorded by Corvis ST [28, 29]. Thus, the model takes into account, non-linear elastic corneal properties as well as extra-corneal properties, e.g., eye globe, fat tissue, and muscles [28, 29]. The model has already been applied to healthy patients to investigate the impact of myopia and age [30] and to differentiate between healthy, suspect, and keratoconic eyes [29].

The aim of the current study was to assess corneal stiffening effects of standard cross-linking (S-CXL) and accelerated cross-linking (A-CXL) protocols with the help of basic parameters of the Corvis ST as well as the novel corneal stiffness parameters derived from the deformation process. These parameters describe the bending properties of the cornea caused by the perpendicular indentation from the air puff and were compared to uniaxial tensile properties of stress-strain measurements.

\section{Methods}

\section{Preparation and cross-linking procedure}

Porcine eyes were transported from a slaughterhouse under cool conditions to the clinical laboratory of the Department of Ophthalmology, University Hospital Carl Gustav Carus, TU Dresden, Germany. Seventy-two porcine eye globes were cleaned off the extra-ocular tissue and were separated into four different groups: 1 - control group ( $n=18$ eyes); 2 -standard CXL group ( $n=18$ eyes), $3 \mathrm{~mW} / \mathrm{cm}^{2}$ for $30 \mathrm{~min}$, S-CXL 
( $3 * 30) ; 3$-accelerated CXL group ( $n=18$ eyes), $9 \mathrm{~mW} / \mathrm{cm}^{2}$ for $10 \mathrm{~min}, \mathrm{~A}-\mathrm{CXL}\left(9^{*} 10\right)$; and $4-$ accelerated CXL group ( $n=18$ eyes), $18 \mathrm{~mW} / \mathrm{cm}^{2}$ for $5 \mathrm{~min}$, A-CXL $(18 * 5)$. Subsequently, all eye globes were de-epithelialized using a hockey knife and afterwards soaked in $0.1 \%$ riboflavin solution including $10 \%$ dextran for $10 \mathrm{~min}$. De-epithelialization was done according the "epi-off" CXL procedure that was described by Wollensak et al. [3]. The control group was only used for comparison between the CXL protocols in stressstrain measurements. However, they were also deepithelialized and soaked in riboflavin solution to avoid different hydration conditions of the corneas.

For air-puff tonometry, the eye globes were mounted in a specially manufactured silicon case and adjusted in front of the Corvis ST. The superior-inferior meridian of the cornea was aligned to the 2-dimensional image of the Corvis ST, which was consistent to subsequent stressstrain measurement. The measurement was performed twice on each eye while they were plugged by a manometer, which was calibrated using a miniature pressure probe (Keller AG, Winterthur, Switzerland). In Fig. 1, the experimental set-up is drawn schematically. The manometer consisted of a reversed bottle containing water and was connected to the eye globe by a tube. A difference between the water surface within the bottle and the mounted eye amounted $27 \mathrm{~cm}$, which ensured a constant intraocular pressure of $20 \mathrm{mmHg}$ during the whole procedure. Following this, the CXL procedure was performed on groups 2, 3, and 4 using the CXL system (CCL Vario, Peschke Trade GmbH, Huenenberg, Switzerland; Fig. 2). The corneas were irradiated with a diameter of $11 \mathrm{~mm}$ and different intensities of irradiance $\left(3 \mathrm{~mW} / \mathrm{cm}^{2}, 9 \mathrm{~mW} / \mathrm{cm}^{2}\right.$, and $18 \mathrm{~mW} / \mathrm{cm}^{2}$ ). Each eye of the CXL group was measured before (pre-treatment) and after CXL (posttreatment) using the Corvis ST. The corneal surface was wetted using a water-soaked sponge releasing the air puff automatically. The complete workflow is shown in Fig. 3.

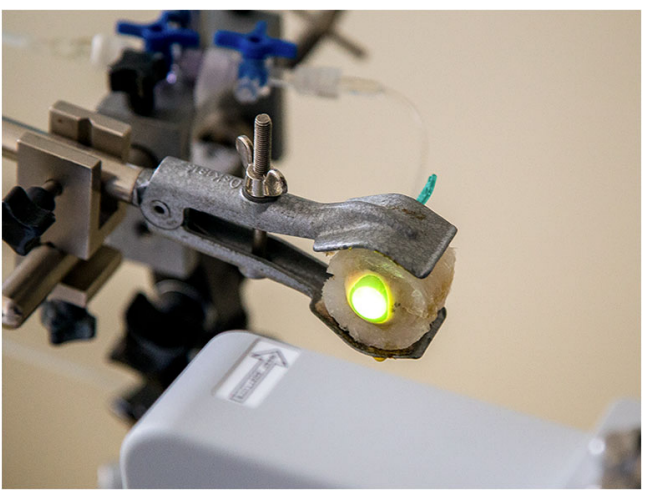

Fig. 2 CXL device (CCL Vario) in front of the porcine eye globe mounted in a silicone case

\section{Stress-strain measurement}

The stress-strain measurement was performed on all groups using the microcomputer-controlled material tester (MINIMAT, Rheometric Scientific GmbH, Bensheim, Germany). Corneal strips $(5 \times 14 \mathrm{~mm})$ were extracted by a double-bladed scalpel in superior-inferior direction and used for stress-strain measurements. After that, the corneal strips were clamped into the device and preloaded $\left(0.02 \mathrm{~N} / \mathrm{m}^{2}\right)$ with a length of around $7 \mathrm{~mm}$ (Fig. 4). Each corneal strip was elongated by increasing the load from 0.02 to $3 \mathrm{~N}$ with a velocity of $2 \mathrm{~mm} / \mathrm{min}$. First, tensile stiffness (K[ts]) was calculated from the slope of a load/force to an extension plot by the material tester software. Second, the slope of stress-strain was exported to a spreadsheet file.

\section{Measurement of dynamic corneal response parameters and waveform-derived corneal stiffness parameters}

The Corvis ST (Corneal Visualization Scheimpflug Technology; Fig. 5) is known to be a dynamic Scheimpflug analyzer that records the corneal deformation process with an
Fig. 1 Experimental set-up of Corvis ST and manometer to ensure constant IOP conditions of $20 \mathrm{mmHg}$




72 fresh enucleated and de-epithelialized porcine eyes

soaked in $0.1 \%$ riboflavin solution ( $10 \%$ dextran) for

$10 \mathrm{~min}$

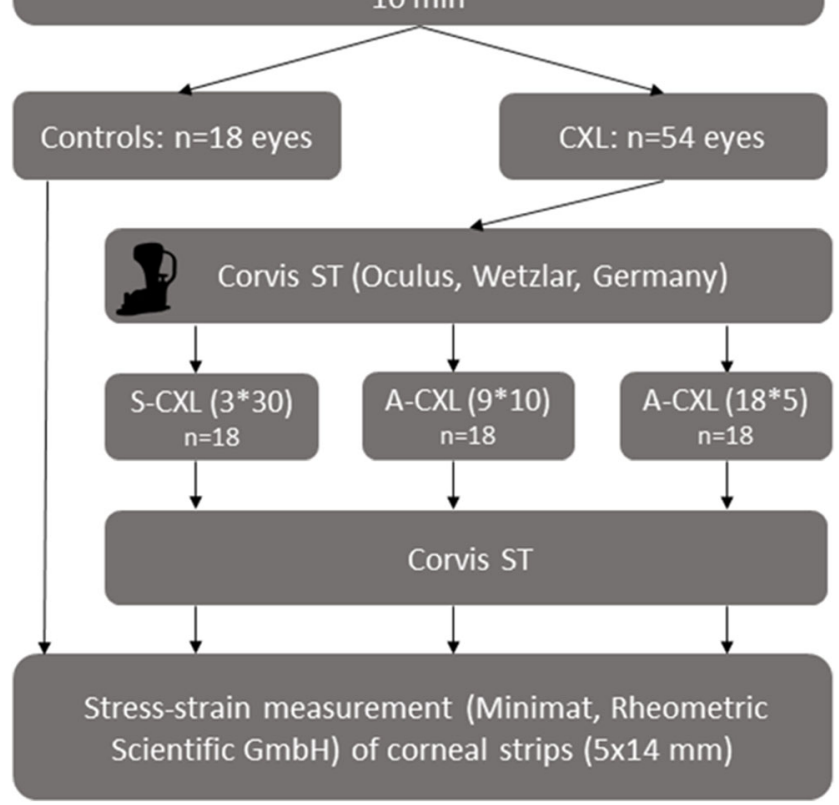

Fig. 3 Flowchart of experimental workflow

ultra-high-speed camera (4300 frames per second). The cornea is forced from its initial convex shape into a first applanation state (A1), followed by a concave phase until the highest concavity (HC) is reached and recovery to the initial state occurs (Fig. 5, center), passing the second applanation state (A2) [20]. The observed corneal diameter accounts for $8 \mathrm{~mm}$. Different dynamic corneal response (DCR) parameters were released during device development. There are basic parameters, e.g., deformation (DA) and deflection amplitude (DeflA), that have been described in previous studies $[20,31]$. The latest major update released new keratoconus-associated DCR parameters and a biomechanical corrected intraocular pressure (bIOP) that is less influenced by age, central corneal thickness (CCT), and other DCR parameters [32]. As bIOP was developed for human eyes, it was

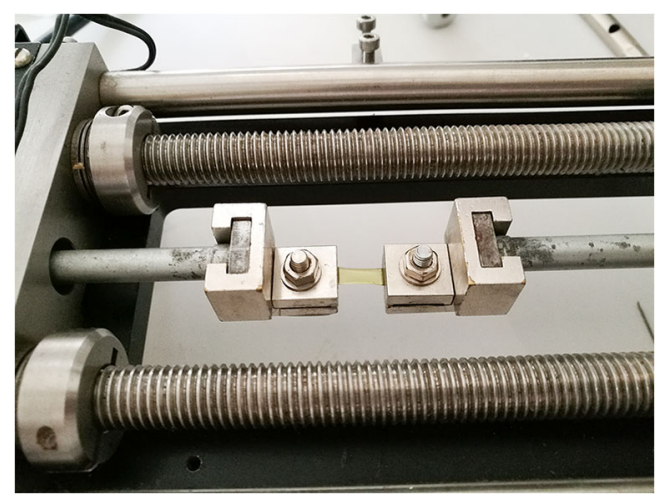

Fig. 4 Strip of corneal tissue mounted by clamps of the extensometer during stress-strain measurement excluded from further analysis because of the use of porcine eyes in our study. Further, maximal inverse radius $(1 / R)$ is the radius at highest concavity (HC-R) where the integrated radius defines the sum of the reciprocal curvature $(1 / R)$ between $\mathrm{A} 1$ and A2 [22]. Another parameter is DA ratio that illustrates the ratio of peripheral (at $1 \mathrm{~mm}$ and $2 \mathrm{~mm}$ ) and central deformation [22]. Further, there is a stiffness parameter (SP A1) calculated at A1 which is described as the difference of adjusted external pressure and bIOP divided by A1 deflection amplitude [23].

Mean corneal stiffness (Kc[mean]) and constant corneal stiffness (Kc[linear]) were calculated from raw data of Corvis ST using a novel analytical model [29, 30]. $\mathrm{Kc}[\mathrm{mean}]$ describes the non-linear elastic behavior, whereas $\mathrm{Kc}$ [linear] describes linear elastic behavior of the corneal tissue. The model was solved using non-linear least square technique MATLAB R2013a (MathWorks, Inc., USA).

\section{Statistical analysis}

Statistical analysis was performed using Excel (2016, Microsoft Corp.) and SPSS (version 25, IBM Corp.). Normal distribution was confirmed by Shapiro-Wilk test and $Q-Q$ plots. For multiple comparisons between all groups at one time level (baseline data, stress-strain data), one-way analysis of variance (ANOVA) with Bonferroni post hoc correction was used. The measurements were analyzed as repeated measurements that were evaluated by linear mixed models. Further, changes in corneal thickness before and after treatment were considered as covariate. A $P$ value less than 0.05 was considered statistically significant.

\section{Results}

\section{Stress-strain extensometry}

According to the workflow, stress-strain measurements were performed after CXL treatment for groups 2, 3, and 4. The tissue of group 1 was soaked in riboflavin and did not obtain CXL. Therefore, it was considered as the control group. Corneal thickness, which was measured by ultrasound pachymetry in corneal strips before stress-strain measurement, showed significantly higher values in the control group compared to A-CXL $(18 * 5)(P=0.026)$, whereas no other significances were observed (all $P>0.05$, Table 1).

Pre-measurement length adjustment of corneal strips was $7 \mathrm{~mm}$ in all groups. There were no significant differences between the four groups, although A-CXL $(18 * 5)$ had slightly longer clamped strips (all $P>0.05$ ). The bar graph of stressstrain measurements (Fig. 6) showed that stress was significantly higher in S-CXL $(3 * 30)$ compared to the controls in all parts of the strain (all $P<0.05$ ). Also, stress was significantly 
Corvis ST

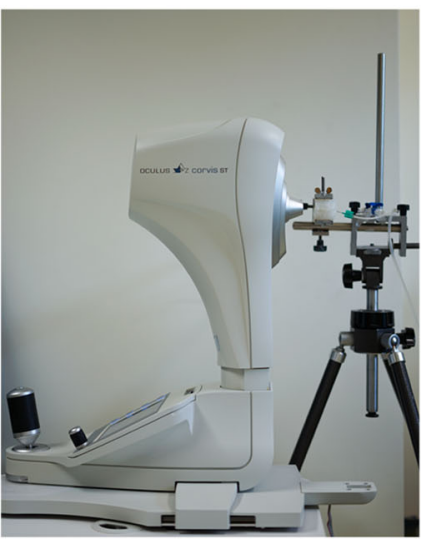

pre treatment S-CXL $(3 * 30)$

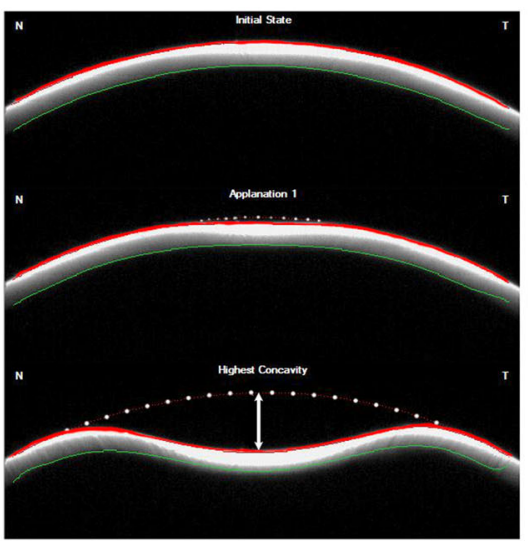

post treatment S-CXL(3*30)

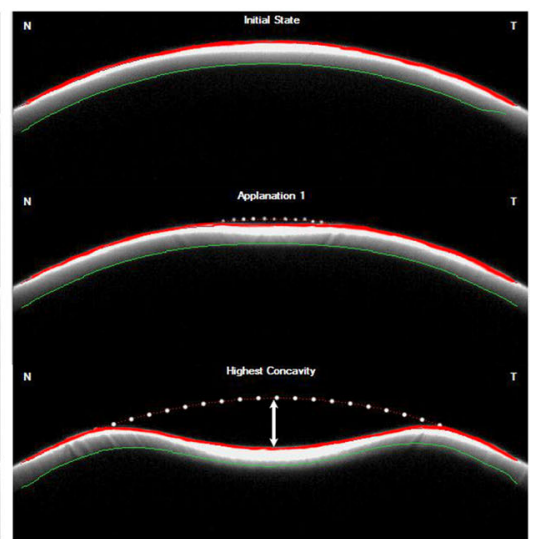

Fig. 5 Corvis ST device in laboratory environment (left) and Scheimpflug images from pre-treatment (center) and post-treatment measurement (right) under constant IOP conditions of $20 \mathrm{mmHg}$. Dotted line represents initial anterior corneal shape. Line with arrows represents deflection amplitude

higher for S-CXL $(3 * 30)$ than A-CXL $(18 * 5)$ at $4 \%, 6 \%$, and $8 \%$ strain (all $P<0.05$ ), but not at $2 \%$ and $10 \%$. A significantly higher stress for S-CXL $(3 * 30)$ compared to A-CXL $(9 * 10)$ could only be observed at $6 \%$ strain $(P<0.05)$. For A-CXL $\left(9^{*} 10\right)$, there was a significantly higher stress at $4 \%, 6 \%, 8 \%$, and $10 \%$ strain compared to controls, whereas no increased stress was found in comparison to A-CXL $(18 * 9)$. Further, stress was significantly higher for A-CXL $(18 * 5)$ than the controls at $8 \%$ and $10 \%$ strain.

Tensile stiffness (K[ts]) was derived from the load to the extension plot by calculating the slope in defined points of extension. The mean extensions that correspond to a strain of $2 \%, 4 \%, 6 \%, 8 \%$, and $10 \%$ were $0.15 \mathrm{~mm}$, $0.29 \mathrm{~mm}, 0.44 \mathrm{~mm}, 0.58 \mathrm{~mm}$, and $0.72 \mathrm{~mm}$, respectively. The results are presented in Table 2. K[ts] was significantly higher for S-CXL $(3 * 30)$ compared to controls $(P<0.05$ in all points), A-CXL $(9 * 10)(P<0.05$ between 0.15 and $0.44 \mathrm{~mm})$, and A-CXL $(18 * 5)(P<0.05$ between 0.15 and $0.44 \mathrm{~mm})$. In longer extensions, A-CXL $(9 * 10)$ and A-CXL $(18 * 5)$ showed significantly higher $\mathrm{K}[\mathrm{ts}]$ than controls. However, they did not differ from each other in all points $(P=1.0)$.

\section{Corvis ST and corneal stiffness}

The CXL groups did not differ in pre-treatment values for CVS-IOP, CVS-CT, SP A1, Kc[mean], and Kc[linear] (all $P>0.05$, Table 3$)$. The alterations in DCR parameters are presented in Table 4 . For S-CXL (3*30), a highly significant increase was observed in CVS-IOP, DA ratio $2 \mathrm{~mm}$, and SP A1 (all $P<0.05$ ). A1 velocity, deflection amplitude, and integrated radius were decreased after treatment (all $P<0.05$ ). Additionally, corneal thickness measured by Corvis ST (CVS-CT) was decreased $(P=0.001)$. Similar results were observed for A-CXL $\left(9^{*} 10\right)$ and A-CXL $(18 * 5)$, whereby DA ratio $2 \mathrm{~mm}$ did not change significantly for A-CXL (9*10) (all $P=0.062$ ). By considering the reduction of CVS$\mathrm{CT}$ during the procedure as covariates in linear mixed models, no effect on above-mentioned results was observed. Concerning corneal stiffness parameter $\mathrm{Kc}$ [mean] and $\mathrm{Kc}$ [linear], a significant increase was shown for all groups. Adjusting CVS-CT, $\mathrm{Kc}[$ mean] was not significantly increased for A-CXL $(18 * 5)$. Comparing the pre- and post-treatment differences between the CXL protocols (Table 5), CVS-IOP increased significantly more in S-CXL $(3 * 30)$ than in A-CXL

Table 1 Descriptive analysis of corneal strips of porcine eyes concerning stress-strain measurement. Significance is marked in italics

\begin{tabular}{|c|c|c|c|c|c|c|c|}
\hline & Controls $(n=18)$ & $\begin{array}{l}\text { S-CXL }(3 * 30) \\
(n=18)\end{array}$ & $\begin{array}{l}\text { A-CXL }(9 * 10) \\
(n=18)\end{array}$ & $\begin{array}{l}\text { A-CXL }(18 * 5) \\
(n=18)\end{array}$ & $P$ value $^{1}$ & $P$ value $^{2}$ & $P$ value ${ }^{3}$ \\
\hline \multicolumn{8}{|l|}{ Mean \pm SD } \\
\hline $\begin{array}{l}\text { Length of sample }[\mathrm{mm}] \\
\text { by a preload of } 0.02 \mathrm{~N} / \mathrm{m}^{2}\end{array}$ & $7.18 \pm 0.2$ & $7.02 \pm 0.19$ & $7.18 \pm 0.35$ & $7.28 \pm 0.41$ & 0.720 & 1.0 & 1.0 \\
\hline Corneal thickness [mm] & $0.74 \pm 0.09$ & $0.69 \pm 0.09$ & $0.69 \pm 0.07$ & $0.67 \pm 0.06$ & 0.265 & 0.253 & 0.026 \\
\hline
\end{tabular}

$A-C X L$ accelerated CXL, $S-C X L$ standard CXL

${ }^{1} P$ value between controls and S-CXL $(3 * 30)$

${ }^{2} P$ value between controls and A-CXL $(9 * 10)$

${ }^{3} P$ value between controls and A-CXL $(18 * 5)$ 
Fig. 6 Stress-strain bar plot of extensometer measurements in different CXL protocols. $* P<0.05$ between $3 \mathrm{~mW}$ and controls. ${ }^{+} P<0.05$ between $9 \mathrm{~mW}$ and controls. ${ }^{\#} P<0.05$ between $3 \mathrm{~mW}$ and $18 \mathrm{~mW}$. $\sim P<0.05$ between $3 \mathrm{~mW}$ and $9 \mathrm{~mW} .{ }^{\circ} \mathrm{P}<0.05$ between $18 \mathrm{~mW}$ and controls

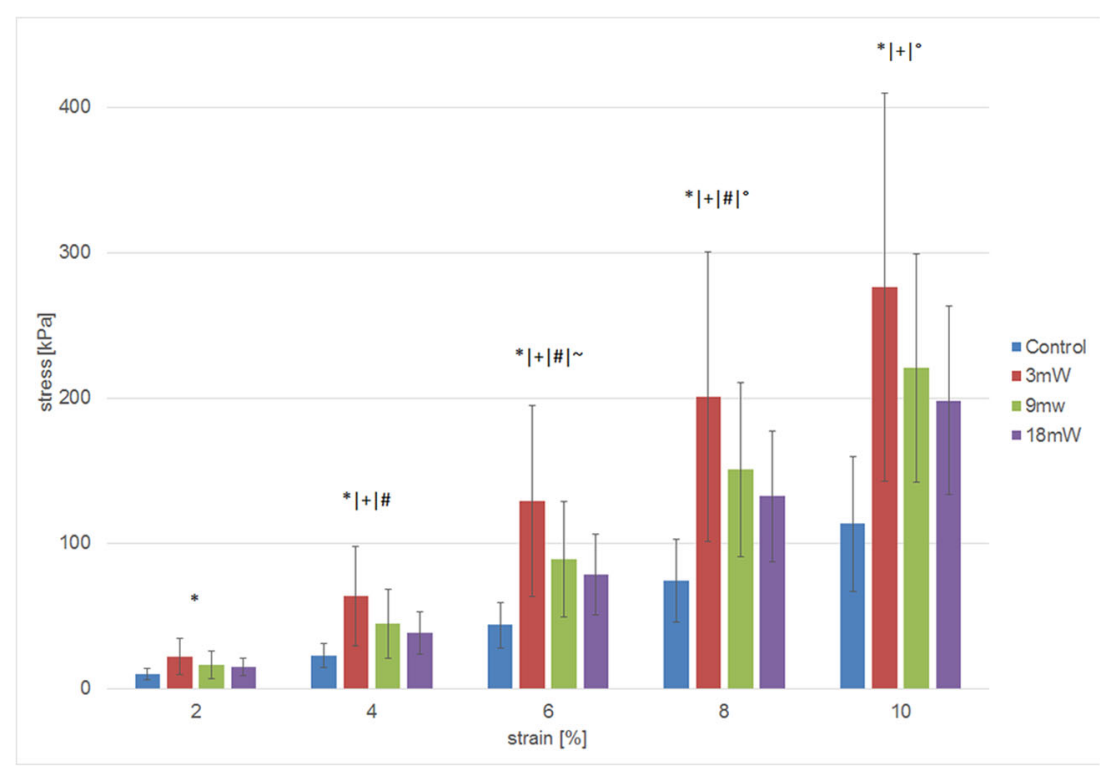

$(9 * 10)$ and A-CXL $(18 * 5)$ as well as in A-CXL $(9 * 10)$ rather than in A-CXL $(18 * 5)$ (all $P<0.05)$. The increase after SCXL(3*30) was significantly higher in DA ratio $2 \mathrm{~mm}$, deflection amplitude, and SP A1 compared to either A-CXL $(9 * 10)$ or A-CXL $(18 * 5)$ or both (all $P<0.05)$. There was no statistical significance between the CXL groups concerning the pre- and post-treatment differences in integrated radius and $\mathrm{Kc}[\mathrm{mean}]$ and $\mathrm{Kc}[$ linear] $(P>0.05)$.

\section{Discussion}

Corneal cross-linking using riboflavin and ultraviolet-A light is an established treatment method to halt progression in keratoconus. It is defined as a photochemical process causing cross-links in corneal tissue, which proceeds mainly intra- and intermolecularly at the fibrils' surface and within the cores of proteoglycan, in the area between the fibrils [33]. Several experimental studies confirmed the stiffening effects of corneal tissue $[1,8,11,34]$. In vivo, the efficacy of CXL is detected by stabilization or flattening of keratometry values of corneal topography in short-term and long-term results $[6,35,36]$. Until now, an assessment of changes in biomechanical properties in vivo, after CXL, could only be performed by waveform-derived parameters (peak 1 and peak 2) using ORA $[16,37,38]$. Sedaghat and co-workers have shown alterations in DCR parameters after CXL, whereas no changes were observed for $\mathrm{CH}$ and $\mathrm{CRF}$ [27]. Further, Vinciguerra and co-workers have shown significant alterations in DCR parameters by the Corvis ST in preliminary results [26].

The aim of this study was to evaluate alterations in corneal biomechanical properties after application of CXL in porcine eyes using stress-strain extensometry and air-puff tonometry. This study has compared three CXL protocols, which are used

Table 2 Comparison of tensile stiffness (K[ts]) derived from load to extension plot. Significance is marked in italics

\begin{tabular}{lllllll}
\hline & $N$ & $\mathrm{~K}[\mathrm{ts}]$ at $0.15 \mathrm{~mm}$ & $\mathrm{~K}[\mathrm{ts}]$ at $0.29 \mathrm{~mm}$ & $\mathrm{~K}[\mathrm{ts}]$ at $0.44 \mathrm{~mm}$ & $\mathrm{~K}[\mathrm{ts}]$ at $0.58 \mathrm{~mm}$ & $\mathrm{~K}[\mathrm{ts}] \mathrm{at} 0.72 \mathrm{~mm}$ \\
\hline Controls & 18 & $231.2 \pm 113.1$ & $425.3 \pm 198.7$ & $650.6 \pm 282.8$ & $936.0 \pm 428.6$ & $1164.5 \pm 533.3$ \\
S-CXL $(3 * 30)$ & 18 & $828.6 \pm 512.8$ & $1440.5 \pm 875.6$ & $1958.9 \pm 1062.1$ & $2135.3 \pm 997.2$ & $1975.9 \pm 803.8$ \\
$P$ value (S-CXL $(3 * 30)-$ controls) & & $<0.001$ & $<0.001$ & $<0.001$ & $<0.001$ & 0.03 \\
A-CXL $(9 * 10)$ & 18 & $472.7 \pm 346.1$ & $896.0 \pm 403.7$ & $1264.6 \pm 574.9$ & $1676.4 \pm 624.8$ & $1747.7 \pm 637.3$ \\
$P$ value (A-CXL $(9 * 10)-$ controls) & & 0.204 & 0.052 & 0.046 & 0.011 & 0.062 \\
$P$ value (A-CXL $(9 * 10)-$ S-CXL $(3 * 30))$ & & 0.013 & 0.016 & 0.017 & 0.285 & 1.0 \\
A-CXL $(18 * 5)$ & 18 & $489.2 \pm 228.9$ & $823.5 \pm 352.2$ & $1325.9 \pm 512.2$ & $1706.1 \pm 539.4$ & $1838.1 \pm 650.0$ \\
$P$ value (A-CXL $(18 * 5)-$ controls) & & 0.143 & 0.152 & 0.021 & 0.007 & 0.020 \\
$P$ value (A-CXL $(18 * 5)-$ S-CXL $(3 * 30))$ & & 0.020 & 0.004 & 0.037 & 0.379 & 1.0 \\
$P$ value (A-CXL $(18 * 5)-$ A-CXL $(9 * 10))$ & 1.0 & 1.0 & 1.0 & 1.0 \\
\hline
\end{tabular}

$A-C X L$ accelerated CXL, $C X L$ corneal cross-linking, $K[t s]$ tensile stiffness in $(\mathrm{N} / \mathrm{m}), S-C X L$ standard CXL 
Table 3 Descriptive analysis of pre-treatment DCR parameters between CXL groups

\begin{tabular}{llllcc}
\hline & $\begin{array}{l}\text { S-CXL }(3 * 30) \\
(n=18)\end{array}$ & $\begin{array}{l}\text { A-CXL }(9 * 10) \\
(n=18)\end{array}$ & $\begin{array}{l}\text { A-CXL }(18 * 5) \\
(n=18)\end{array}$ & $P$ value $^{1}$ & $P$ value $^{2}$ \\
\hline Mean \pm SD & & & & & \\
CVS-IOP $[\mathrm{mmHg}]$ & $9.89 \pm 0.95$ & $10.42 \pm 0.93$ & $10.11 \pm 0.9$ & 0.385 & 1.0 \\
CVS-CT $[\mu \mathrm{m}]$ & $699.7 \pm 74.5$ & $692.4 \pm 58.8$ & $697.7 \pm 62.7$ & 0.756 & 0.930 \\
SP A1 $[\mathrm{mmHg} / \mathrm{mm}]$ & $75.46 \pm 9.4$ & $80.21 \pm 9.08$ & $76.61 \pm 10.46$ & 0.714 & 1.0 \\
Kc[mean] $[\mathrm{N} / \mathrm{m}]$ & $78.08 \pm 11.97$ & $85.88 \pm 10.74$ & $82.93 \pm 10.07$ & 0.285 & 1.0 \\
Kc[linear] $[\mathrm{N} / \mathrm{m}]$ & $81.58 \pm 11.59$ & $89.13 \pm 8$ & $84.16 \pm 9.6$ & 0.192 & 1.0 \\
\hline
\end{tabular}

$A-C X L$ accelerated CXL, CVS-CT corneal thickness measured by Corvis ST, CVS-IOP uncorrected IOP measured by Corvis ST, $C X L$ corneal crosslinking, $I O P$ intraocular pressure, $K c$ corneal stiffness derived from waveform analysis, $S$ - $C X L$ standard CXL, SP A1 stiffness parameter at 1 st applanation

${ }^{1} P$ value between S-CXL $(3 * 30)$ and A-CXL $(9 * 10)$

${ }^{2} P$ value between S-CXL $(3 * 30)$ and A-CXL $(18 * 5)$

${ }^{3} P$ value between A-CXL $(9 * 10)$ and A-CXL $(18 * 5)$

in clinical practice, S-CXL ( $3 * 30)$, A-CXL $(9 * 10)$, and ACXL $(18 * 5)$. The modification of intensity and time is according the Bunsen-Roscoe Law, while the total energy remains constant $\left(5.4 \mathrm{~J} / \mathrm{cm}^{2}\right)$. According to experimental methods of this study, all specimens were soaked in riboflavin solution containing $10 \%$ dextran to avoid corneal swelling. It is known that biomechanical properties of the cornea depend on corneal hydration, whereas hydrated corneas have weaker tensile properties than dehydrated corneas [39]. In case of CXL, Hatami-Marbini and Jayaram have shown that corneal stiffening depended on pre-treatment corneal hydration as well; however, the improvement of tensile properties after CXL was not different, if mechanical tests were done under the same hydration conditions [40]. Further, riboflavin with dextran tends to decrease corneal thickness during CXL treatment [41]. Therefore, we have decided to use a dextran concentration of $10 \%$. Nonetheless, corneal thickness alterations were considered for stress-strain as well as Corvis ST measurements.

In our study, we found an increased stiffness for S-CXL ( $3 * 30)$, A-CXL $(9 * 10)$, and A-CXL $(18 * 5)$ at $10 \%$ strain compared to controls. These results are similar to investigations of Schumacher et al., Krueger et al., and Wernli et al. The latter found significant stiffening up to $45 \mathrm{~mW} / \mathrm{cm}^{2}$ [8]. In contrast, Hammer et al. showed that A-CXL $(18 * 5)$ were not significantly stiffer than controls [11]. The reason for the different outcomes might be various experimental set-ups that are summarized in Table 6. Additionally, inflation tests performed by Bao et al. have shown that the shorter the irradiation time is, the lower is the increase in stiffening the tissue [42]. However, these results were observed at $4 \%$ strain that is equal approximately to physiological strain of the cornea under normal IOP conditions [42]. Our results confirm these findings with regard to the stress-strain plot. At $2 \%$ strain, corneas treated with S-CXL $(3 * 30)$ were significant stiffer than the controls. At 4\% strain, both S-CXL (3*30) and ACXL $(9 * 10)$ were stiffer than the controls, whereas S-CXL
(3*30) was stiffer than A-CXL (18*5). Nevertheless, it can be assumed that accelerated protocols are effective up to $9 \mathrm{~mW} / \mathrm{cm}^{2}$ with a total intensity of $5.4 \mathrm{~J} / \mathrm{cm}^{2}$. Further, tensile stiffness $(\mathrm{K}[\mathrm{ts}])$ was calculated from load to extension plots and showed similar results as in stress-strain. However, K[ts] was not significantly higher for A-CXL (9*10) in comparison to controls at $0.72 \mathrm{~mm}$ (equal to $10 \%$ strain), whereas a significant stiffening occurred at $0.58 \mathrm{~mm}$ (equal to $8 \%$ strain).

In this study, we examined the differences between the preand post-treatment of certain DCR and stiffness parameters (Kc[mean, Kc[linear]) using the complete porcine eye globe that was mounted in a special silicon case under a watercolumn-controlled intraocular pressure of $20 \mathrm{mmHg}$. This means that our experimental set-up took into account effects of ocular tissue (e.g., sclera) of the whole eye globe [43]. The changes in DCR parameters and $\mathrm{Kc}$ [mean/linear] confirmed the stiffening effect of CXL in all used protocols. First, we observed a significant decrease of corneal thickness (CT) measured by Corvis before and after CXL. Thus, each parameter was separately analyzed, considering the impact of CT. In all three CXL protocols, the CVS-IOP was measured to be significantly higher after treatment, while the IOP was adjusted to a constant at $20 \mathrm{mmHg}$ over the whole procedure. This indicates that elevated measured IOP after CXL showed a stiffer behavior of the eye wall, especially the cornea. An increased IOP in Goldmann applanation tonometry after CXL was reported in vivo by Kymionis et al. and Kasumovic et al. [44, 45]. The deflection amplitude was significantly decreased in all protocols, even though CT was adjusted. This leads to the assumption that the cornea is less deformable against the air puff. Further, the significant reduction of maximal inverse radius $(1 / R)$ indicates a flattened concave radius at highest concavity (HC-R) in all protocols. In vivo, Sedaghat et al. and Hashemi et al. have also observed an increased HC-R after CXL in standard [27] and accelerated protocols [46], respectively. Following this, values of integrated (inverse) radius were 


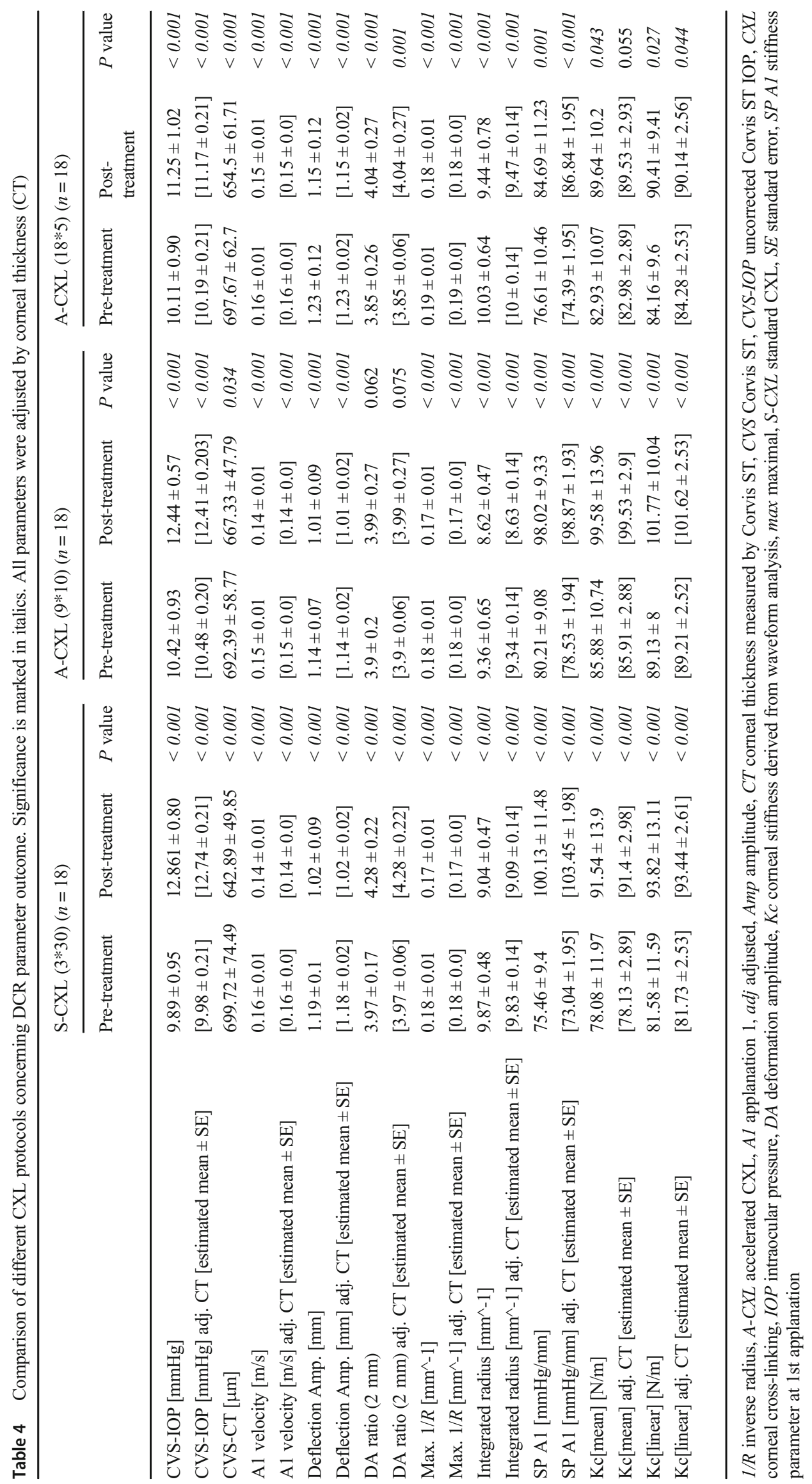


Table 5 Comparison of differences between pre- and post-treatment in performed CXL protocols. Significance is marked in italics

\begin{tabular}{|c|c|c|c|c|c|c|}
\hline Parameter & $\begin{array}{l}\text { Difference of } \\
\text { S-CXL }(3 * 30)\end{array}$ & $\begin{array}{l}\text { Difference of } \\
\text { A-CXL }(9 * 10)\end{array}$ & $\begin{array}{l}\text { Difference of } \\
\text { A-CXL }(18 * 5)\end{array}$ & $\begin{array}{l}P \text { value } \\
(3 * 30-9 * 10)\end{array}$ & $\begin{array}{l}P \text { value } \\
(3 * 30-18 * 5)\end{array}$ & $\begin{array}{l}P \text { value } \\
(9 * 10-18 * 5)\end{array}$ \\
\hline CVS-IOP [mmHg] & $+2.97 \pm 0.76$ & $+2.03 \pm 0.79$ & $+1.14 \pm 1.04$ & 0.006 & 0.000 & 0.011 \\
\hline CVS-CT $[\mu \mathrm{m}]$ & $-56.83 \pm 55.13$ & $-25.06 \pm 58.8$ & $-43.17 \pm 25.43$ & 0.169 & 1.000 & 0.812 \\
\hline A1 velocity $[\mathrm{m} / \mathrm{s}]$ & $-0.02 \pm 0.01$ & $-0.01 \pm 0.01$ & $-0.01 \pm 0.01$ & 0.973 & 0.258 & 1.000 \\
\hline Deflection Amp [mm] & $-0.17 \pm 0.04$ & $-0.14 \pm 0.05$ & $-0.09 \pm 0.09$ & 0.575 & 0.001 & 0.042 \\
\hline DA ratio $2 \mathrm{~mm}[\mathrm{~mm}]$ & $+0.31 \pm 0.16$ & $+0.09 \pm 0.23$ & $+0.19 \pm 0.23$ & 0.009 & 0.287 & 0.496 \\
\hline Integrated radius $\left[\mathrm{mm}^{\wedge}-1\right]$ & $-0.83 \pm 0.36$ & $-0.74 \pm 0.36$ & $-0.59 \pm 0.55$ & 1.000 & 0.344 & 0.912 \\
\hline SP A1 $[\mathrm{mmHg} / \mathrm{mm}]$ & $+24.66 \pm 9.27$ & $+17.81 \pm 10.07$ & $+8.08 \pm 8.78$ & 0.100 & 0.000 & 0.009 \\
\hline $\mathrm{Kc}[\mathrm{mean}][\mathrm{N} / \mathrm{m}]$ & $+13.46 \pm 18.63$ & $+13.7 \pm 8.64$ & $+6.7 \pm 11.42$ & 1.000 & 0.423 & 0.383 \\
\hline $\mathrm{Kc}[$ linear $][\mathrm{N} / \mathrm{m}]$ & $+12.23 \pm 15.83$ & $+12.64 \pm 6.45$ & $+6.26 \pm 10.25$ & 1.000 & 0.376 & 0.306 \\
\hline
\end{tabular}

A-CXL accelerated CXL, A1 applanation 1, adj adjusted, Amp amplitude, CT corneal thickness measured by Corvis ST, CVS Corvis ST, CVS-IOP uncorrected Corvis ST IOP, CXL corneal cross-linking, IOP intraocular pressure, $D A$ deformation amplitude, $K c$ corneal stiffness derived from waveform analysis, $S$-CXL standard CXL, $S E$ standard error, $S P A 1$ stiffness parameter at 1st applanation

significantly decreased in our results for all protocols. This was also shown in vivo for standard [27] and accelerated protocols $[26,47,48]$. Additionally, in recent studies, the deformation amplitude ratio (DA ratio) was decreased after CXL in different cohorts, whereby not all data showed significance $[26,27,47$, 48]. These results are in line with the definition, which states that higher values of the DA ratio are associated with softer corneas [22]. Therefore, a decrease of the DA ratio is expected after CXL. In contrast, our experimental results showed that the DA ratio $(2 \mathrm{~mm})$ increases in all protocols after CXL, even though the deformation amplitude (data not shown) and deflection amplitude were reduced. Coming back to its definition, this might explain that CXL led to less deformation of the peripheral cornea and, thus, a higher DA ratio. On the other hand, the higher thickness and the "normal" peripheral thickness profile of the porcine eye model possibly distorted these results.

SP A1 is the stiffness parameter calculated for reaching the first applanation (A1), which was significantly increased in standard and accelerated protocols. In literature, there are some in vivo reports that have shown increased values as well but not all were significant $[26,27,47,48]$. Corneal stiffness expressed in newtons per meter $(\mathrm{Kc}[\mathrm{mean}], \mathrm{Kc}[$ linear] $)$ showed a similar increase in S-CXL and A-CXL $(9 * 10)$, whereas ACXL $(18 * 5)$ was less pronounced compared to SP A1.

Stress-strain extensometry confirmed stiffening of the corneal tissue for S-CXL $(3 * 30)$ and A-CXL $(9 * 10)$ in physiological conditions (at 4\% strain) and for all CXL protocols at $10 \%$ strain. Previously, the same specimens were examined with the Corvis ST to obtain alterations before and after CXL in DCR parameters. Therefore, it can be assumed that the corneal stiffening induced by the CXL procedure has affected the biomechanical properties of the cornea. Stiffness is described as the resistance of an object against a deformation [23]. A higher stiffness of the cornea implicates a less deformation to an applied force to the cornea. Hence, a greater force is necessary to deform the cornea, e.g., in IOP measurements, whereby the IOP is overestimated [23]. In our study, we have applied a constant IOP to the eye globe during the whole procedure. Our results confirmed corneal stiffening by overestimation of CVS-IOP after CXL treatment under unchanged environmental conditions. Further, velocity until 1st applanation and deflection amplitude was lower after CXL

Table 6 Comparison of experimental ex vivo investigations concerning induced corneal stiffness by cross-linking with riboflavin solution and ultraviolet-A irradiation in porcine eyes

\begin{tabular}{llllll}
\hline Group & Specimen & Irradiation area & Test and sample dimensions & UV intensity & Chromophore (centration) \\
\hline Schumacher et al. [51] & Porcine & $9 \mathrm{~mm}$ & Uniaxial, $7 \mathrm{~mm} \times 1 \mathrm{~mm}$ & $3 \mathrm{~mW}$ and $9 \mathrm{~mW}$ & $0.1 \%$ riboflavin with 20\% dextran \\
Krüger et al. [52] & Porcine & n.a. & Uniaxial, $8 \mathrm{~mm} \times 5 \mathrm{~mm}$ & $2 \mathrm{~mW}$ to $15 \mathrm{~mW}$ & $0.1 \%$ riboflavin with CMC \\
Wernli et al. [8] & Porcine & $\sim 9 \mathrm{~mm}$ & Uniaxial, $7 \mathrm{~mm} \times 5 \mathrm{~mm}$ & $3 \mathrm{~mW}$ to $90 \mathrm{~mW}$ & $0.1 \%$ riboflavin \\
Hammer et al. [11] & Porcine & $11.3 \mathrm{~mm}$ & Uniaxial, $10 \mathrm{~mm} \times 5 \mathrm{~mm}$ & $3 \mathrm{~mW}$ to $15 \mathrm{~mW}$ & $0.1 \%$ riboflavin \\
Kling et al. [34] & Porcine & n.a. & 2-dimensional, $10 \mathrm{~mm}$ & $1.5 \mathrm{~mW}$ and $3 \mathrm{~mW}$ & $0.1 \%$ riboflavin \\
Bao et al. [42] & Japanese rabbits & $9 \mathrm{~mm}$ & Inflation tests & $3 \mathrm{~mW}$ to $90 \mathrm{~mW}$ & $0.22 \%$ riboflavin \\
\hline
\end{tabular}

CMC carboxymethylcellulose, n.a. not applicable 
due to higher stiffness and greater resistance against the air puff. However, deflection amplitude and CVS-IOP have a strong relationship and should be used with caution as single parameter to evaluate alterations in biomechanical properties after CXL in clinical practice [26,32]. Also, the shape of the inward movement of the stiffened cornea has been altered; thus far, the maximal $1 / R$ was reduced implying a flatter curvature at $\mathrm{HC}[23,26]$. Therefore, the integrated inverse radius has been reduced as the cornea became stiffer. SP A1, $\mathrm{Kc}[$ mean], and $\mathrm{Kc}$ [linear] are parameters that describe the resistance against deformation (at 1st applanation) and nonlinear as well as linear behavior based on the deformation amplitude of the corneal tissue, respectively. These parameters were observed to be lower in softer eyes like keratoconus $[23,29]$. In our study, they showed higher values after CXL implicating stiffening of the corneal tissue. None of the other DCR parameters were analyzed due to their low importance in clinical practice. Further, DCR parameters that describe the outward movement until 2nd applanation are influenced by viscoelastic properties of the tissue and are not comparable to uniaxial strip extensometry.

Comparing the differences after treatment in all protocols, there is a trend that S-CXL induced a higher change in corneal response to the air puff, than A-CXL protocols. However, significance was only shown in CVS-IOP. Additionally, a higher stiffening effect of S-CXL than A-CXL $(18 * 5)$ was seen in the deflection amplitude and SP A1. Further, the increase of $\mathrm{Kc}[\mathrm{mean}]$ and $\mathrm{Kc}[$ linear $]$ is more pronounced in S-CXL than in A-CXL protocols, whereby no significance was achieved.

The study is limited in the way that only ex vivo porcine eyes were used to evaluate CXL effects with stress-strain measurements and Corvis ST. Previous studies have shown that porcine eyes are less stiff than human donor eyes, measured in corneal [49] and scleral tissue [50]. Another limitation is that it was not possible to use paired eyes of the same pig to compare different CXL protocols.

In conclusion, in this study, we showed that Corvis ST is able to detect effects of corneal cross-linking in the biomechanical behavior of ex vivo porcine eyes, under laboratory conditions. Furthermore, this study investigated commonly used CXL protocols that increase the corneal stiffness, provably by stress-strain measurements. Thus, changes in DCR parameters captured by Corvis ST were validated by stressstrain measurements. Corvis ST might be useful to assess effectiveness of CXL and support topographic and tomographic data during follow-up examinations.

Funding information Open Access funding provided by Projekt DEAL. $\mathrm{RH}, \mathrm{MF}, \mathrm{ES}, \mathrm{FR}$, and ASR were supported by a research grant from the Indo-German Science and Technology Center, Department of Science and Technology, DLR, Germany, and Indo-German Science and Technology Center, Department of Science and Technology, Government of India (grant number: 01DQ15016A). The sponsor had no role in the design or conduct of this research.

\section{Compliance with ethical standards}

Conflict of interest The authors declare that they have no conflict of interest.

Ethical approval All applicable international, national, and institutional guidelines for the care and use of animals were followed.

Open Access This article is licensed under a Creative Commons Attribution 4.0 International License, which permits use, sharing, adaptation, distribution and reproduction in any medium or format, as long as you give appropriate credit to the original author(s) and the source, provide a link to the Creative Commons licence, and indicate if changes were made. The images or other third party material in this article are included in the article's Creative Commons licence, unless indicated otherwise in a credit line to the material. If material is not included in the article's Creative Commons licence and your intended use is not permitted by statutory regulation or exceeds the permitted use, you will need to obtain permission directly from the copyright holder. To view a copy of this licence, visit http://creativecommons.org/licenses/by/4.0/.

\section{References}

1. Spoerl E, Huhle M, Seiler T (1998) Induction of cross-links in corneal tissue. Exp Eye Res 66:97-103. https://doi.org/10.1006/exer.1997. 0410

2. Spoerl E, Seiler T (1999) Techniques for stiffening the cornea. J Refract Surg 15:711-713

3. Wollensak G, Spoerl E, Seiler T (2003) Riboflavin/ultraviolet-ainduced collagen crosslinking for the treatment of keratoconus. Am J Ophthalmol 135:620-627

4. Gomes JA, Tan D, Rapuano CJ, Belin MW, Ambrosio R Jr, Guell JL, Malecaze F, Nishida K, Sangwan VS, Group of Panelists for the Global Delphi Panel of K, Ectatic D (2015) Global consensus on keratoconus and ectatic diseases. Cornea 34:359-369. https://doi. org/10.1097/ICO.0000000000000408

5. Roberts CJ, Dupps WJ Jr (2014) Biomechanics of corneal ectasia and biomechanical treatments. J Cataract Refract Surg 40:991-998. https://doi.org/10.1016/j.jcrs.2014.04.013

6. Wittig-Silva C, Chan E, Islam FM, Wu T, Whiting M, Snibson GR (2014) A randomized, controlled trial of corneal collagen crosslinking in progressive keratoconus: three-year results. Ophthalmology 121:812-821. https://doi.org/10.1016/j.ophtha. 2013.10.028

7. Spoerl E, Mrochen M, Sliney D, Trokel S, Seiler T (2007) Safety of UVA-riboflavin cross-linking of the cornea. Cornea 26:385-389. https://doi.org/10.1097/ICO.0b013e3180334f78

8. Wernli J, Schumacher S, Spoerl E, Mrochen M (2013) The efficacy of corneal cross-linking shows a sudden decrease with very high intensity UV light and short treatment time. Invest Ophthalmol Vis Sci 54:1176-1180. https://doi.org/10.1167/iovs.12-11409

9. Lang PZ, Hafezi NL, Khandelwal SS, Torres-Netto EA, Hafezi F, Randleman JB (2019) Comparative functional outcomes after corneal crosslinking using standard, accelerated, and accelerated with higher total fluence protocols. Cornea https://doi.org/10.1097/ICO. 0000000000001878

10. Wollensak G, Spoerl E, Seiler T (2003) Stress-strain measurements of human and porcine corneas after riboflavin-ultraviolet-Ainduced cross-linking. J Cataract Refract Surg 29:1780-1785

11. Hammer A, Richoz O, Arba Mosquera S, Tabibian D, Hoogewoud F, Hafezi F (2014) Corneal biomechanical properties at different 
corneal cross-linking (CXL) irradiances. Invest Ophthalmol Vis Sci 55:2881-2884. https://doi.org/10.1167/iovs.13-13748

12. Kling S, Remon L, Perez-Escudero A, Merayo-Lloves J, Marcos S (2010) Corneal biomechanical changes after collagen cross-linking from porcine eye inflation experiments. Invest Ophthalmol Vis Sci 51:3961-3968. https://doi.org/10.1167/iovs.09-4536

13. Fontes BM, Ambrosio R Jr, Velarde GC, Nose W (2011) Ocular response analyzer measurements in keratoconus with normal central corneal thickness compared with matched normal control eyes. J Refract Surg 27:209-215. https://doi.org/10.3928/1081597X20100415-02

14. Fontes BM, Ambrosio R Jr, Jardim D, Velarde GC, Nose W (2010) Corneal biomechanical metrics and anterior segment parameters in mild keratoconus. Ophthalmology 117:673-679. https://doi.org/10. 1016/j.ophtha.2009.09.023

15. Schweitzer C, Roberts CJ, Mahmoud AM, Colin J, Maurice-Tison S, Kerautret J (2010) Screening of forme fruste keratoconus with the ocular response analyzer. Invest Ophthalmol Vis Sci 51:24032410. https://doi.org/10.1167/iovs.09-3689

16. Spoerl E, Terai N, Scholz F, Raiskup F, Pillunat LE (2011) Detection of biomechanical changes after corneal cross-linking using ocular response analyzer software. J Refract Surg 27:452457. https://doi.org/10.3928/1081597X-20110106-01

17. Greenstein SA, Fry KL, Hersh PS (2012) In vivo biomechanical changes after corneal collagen cross-linking for keratoconus and corneal ectasia: 1-year analysis of a randomized, controlled, clinical trial. Cornea 31:21-25. https://doi.org/10.1097/ICO. 0b013e31821 eea66

18. Roberts CJ (2014) Concepts and misconceptions in corneal biomechanics. J Cataract Refract Surg 40:862-869. https://doi.org/10. 1016/j.jcrs.2014.04.019

19. Sinjab MM, Cummings AB (2017) Corneal collagen cross linking. Springer International Publishing, Imprint, Springer, Cham

20. Hon Y, Lam AK (2013) Corneal deformation measurement using Scheimpflug noncontact tonometry. Optom Vis Sci 90:e1-e8. https://doi.org/10.1097/OPX.0b013e318279eb87

21. Hong J, Xu J, Wei A, Deng SX, Cui X, Yu X, Sun X (2013) A new tonometer-the Corvis ST tonometer: clinical comparison with noncontact and Goldmann applanation tonometers. Invest Ophthalmol Vis Sci 54:659-665. https://doi.org/10.1167/iovs.12-10984

22. Vinciguerra R, Ambrosio R Jr, Elsheikh A, Roberts CJ, Lopes B, Morenghi E, Azzolini C, Vinciguerra P (2016) Detection of keratoconus with a new biomechanical index. J Refract Surg 32: 803-810. https://doi.org/10.3928/1081597X-20160629-01

23. Roberts CJ, Mahmoud AM, Bons JP, Hossain A, Elsheikh A, Vinciguerra R, Vinciguerra P, Ambrosio R Jr (2017) Introduction of two novel stiffness parameters and interpretation of air puffinduced biomechanical deformation parameters with a dynamic Scheimpflug analyzer. J Refract Surg 33:266-273. https://doi.org/ 10.3928/1081597X-20161221-03

24. Herber R, Ramm L, Spoerl E, Raiskup F, Pillunat LE, Terai N (2019) Assessment of corneal biomechanical parameters in healthy and keratoconic eyes using dynamic bidirectional applanation device and dynamic Scheimpflug analyzer. J Cataract Refract Surg 45:778-788. https://doi.org/10.1016/j.jcrs.2018.12.015

25. Ambrosio R Jr, Lopes BT, Faria-Correia F, Salomao MQ, Buhren J, Roberts CJ, Elsheikh A, Vinciguerra R, Vinciguerra P (2017) Integration of Scheimpflug-based corneal tomography and biomechanical assessments for enhancing ectasia detection. J Refract Surg 33:434 443. https://doi.org/10.3928/1081597X-20170426-02

26. Vinciguerra R, Romano V, Arbabi EM, Brunner M, Willoughby CE, Batterbury M, Kaye SB (2017) In vivo early corneal biomechanical changes after corneal cross-linking in patients with progressive keratoconus. J Refract Surg 33:840-846. https://doi.org/ 10.3928/1081597X-20170922-02
27. Sedaghat MR, Momeni-Moghaddam H, Ambrosio R Jr, Roberts CJ, Yekta AA, Danesh Z, Reisdorf S, Khabazkhoob M, Heidari HR, Sadeghi J (2018) Long-term evaluation of corneal biomechanical properties after corneal cross-linking for keratoconus: a 4-year longitudinal study. J Refract Surg 34:849-856. https://doi.org/10. 3928/1081597X-20181012-02

28. Sinha Roy A, Kurian M, Matalia H, Shetty R (2015) Air-puff associated quantification of non-linear biomechanical properties of the human cornea in vivo. J Mech Behav Biomed Mater 48:173182. https://doi.org/10.1016/j.jmbbm.2015.04.010

29. Francis M, Pahuja N, Shroff R, Gowda R, Matalia H, Shetty R, Remington Nelson EJ, Sinha Roy A (2017) Waveform analysis of deformation amplitude and deflection amplitude in normal, suspect, and keratoconic eyes. J Cataract Refract Surg 43:1271-1280. https://doi.org/10.1016/j.jcrs.2017.10.012

30. Matalia J, Francis M, Tejwani S, Dudeja G, Rajappa N, Sinha Roy A (2016) Role of age and myopia in simultaneous assessment of corneal and extraocular tissue stiffness by air-puff applanation. J Refract Surg 32:486-493. https://doi.org/10.3928/1081597X20160512-02

31. Miki A, Maeda N, Ikuno Y, Asai T, Hara C, Nishida K (2017) Factors associated with corneal deformation responses measured with a dynamic Scheimpflug analyzer. Invest Ophthalmol Vis Sci 58:538-544. https://doi.org/10.1167/iovs.16-21045

32. Vinciguerra R, Elsheikh A, Roberts CJ, Ambrosio R Jr, Kang DS, Lopes BT, Morenghi E, Azzolini C, Vinciguerra P (2016) Influence of pachymetry and intraocular pressure on dynamic corneal response parameters in healthy patients. J Refract Surg 32:550-561. https://doi.org/10.3928/1081597X-20160524-01

33. Hayes S, Kamma-Lorger CS, Boote C, Young RD, Quantock AJ, Rost A, Khatib Y, Harris J, Yagi N, Terrill N, Meek KM (2013) The effect of riboflavin/UVA collagen cross-linking therapy on the structure and hydrodynamic behaviour of the ungulate and rabbit corneal stroma. PLoS One 8:e52860. https://doi.org/10.1371/ journal.pone. 0052860

34. Kling S, Hafezi F (2017) Biomechanical stiffening: slow lowirradiance corneal crosslinking versus the standard Dresden protocol. J Cataract Refract Surg 43:975-979. https://doi.org/10.1016/j. jers.2017.04.041

35. Vinciguerra R, Romano MR, Camesasca FI, Azzolini C, Trazza S, Morenghi E, Vinciguerra P (2013) Corneal cross-linking as a treatment for keratoconus: four-year morphologic and clinical outcomes with respect to patient age. Ophthalmology 120:908-916. https:// doi.org/10.1016/j.ophtha.2012.10.023

36. Raiskup F, Theuring A, Pillunat LE, Spoerl E (2015) Corneal collagen crosslinking with riboflavin and ultraviolet-a light in progressive keratoconus: ten-year results. J Cataract Refract Surg 41:4146. https://doi.org/10.1016/j.jcrs.2014.09.033

37. Uzel MM, Koc M, Can C, Polat S, Yilmazbas P, Ileri D (2019) Effect of accelerated corneal crosslinking on ocular response analyzer waveform-derived parameters in progressive keratoconus. Arq Bras Oftalmol 82:18-24. https://doi.org/10.5935/0004-2749.20190003

38. Vinciguerra P, Albe E, Mahmoud AM, Trazza S, Hafezi F, Roberts CJ (2010) Intra- and postoperative variation in ocular response analyzer parameters in keratoconic eyes after corneal cross-linking. J Refract Surg 26:669-676. https://doi.org/10.3928/1081597X20100331-01

39. Hatami-Marbini H, Rahimi A (2014) The relation between hydration and mechanical behavior of bovine cornea in tension. J Mech Behav Biomed Mater 36:90-97. https://doi.org/10.1016/j.jmbbm.2014.03.011

40. Hatami-Marbini H, Jayaram SM (2018) Relationship between initial corneal hydration and stiffening effects of corneal crosslinking treatment. J Cataract Refract Surg 44:756-764. https://doi.org/10. 1016/j.jcrs.2018.01.036

41. Kymionis GD, Kounis GA, Portaliou DM, Grentzelos MA, Karavitaki AE, Coskunseven E, Jankov MR, Pallikaris IG (2009) 
Intraoperative pachymetric measurements during corneal collagen cross-linking with riboflavin and ultraviolet a irradiation. Ophthalmology 116:2336-2339. https://doi.org/10.1016/j.ophtha. 2009.09.018

42. Bao F, Zheng Y, Liu C, Zheng X, Zhao Y, Wang Y, Li L, Wang Q, Chen S, Elsheikh A (2018) Changes in corneal biomechanical properties with different corneal cross-linking irradiances. J Refract Surg 34:51-58. https://doi.org/10.3928/1081597X-20171025-01

43. Metzler KM, Mahmoud AM, Liu J, Roberts CJ (2014) Deformation response of paired donor corneas to an air puff: intact whole globe versus mounted corneoscleral rim. J Cataract Refract Surg 40:888896. https://doi.org/10.1016/j.jcrs.2014.02.032

44. Kymionis GD, Grentzelos MA, Kounis GA, Portaliou DM, Detorakis ET, Magarakis M, Karampatakis VE, Pallikaris IG (2010) Intraocular pressure measurements after corneal collagen crosslinking with riboflavin and ultraviolet $\mathrm{A}$ in eyes with keratoconus. J Cataract Refract Surg 36:1724-1727. https://doi. org/10.1016/j.jcrs.2010.05.016

45. Kasumovic SS, Mavija M, Kasumovic A, Lepara O, Duric-Colic B, Cabric E, Muhamedagic L, Sakovic-Racic A, Jankov M (2015) Intraocular pressure measurements referring to the corneal thickness in keratoconic eyes after corneal crosslinking with riboflavin and ultraviolet a. Med Arch 69:334-338. https://doi.org/10.5455/ medarh.2015.69.334-338

46. Hashemi H, Asgari S, Mehravaran S, Miraftab M, Ghaffari R, Fotouhi A (2017) Corneal biomechanics after accelerated crosslinking: comparison between 18 and $9 \mathrm{~mW} / \mathrm{cm}(2)$ protocols. J Refract Surg 33:558-562. https://doi.org/10.3928/1081597X20170621-04
47. Vinciguerra R, Tzamalis A, Romano V, Arbabi EM, Batterbury M, Kaye SB (2019) Assessment of the association between in vivo corneal biomechanical changes after corneal cross-linking and depth of demarcation line. J Refract Surg 35:202-206. https://doi. org/10.3928/1081597X-20190124-01

48. Hashemi H, Ambrosio R Jr, Vinciguerra R, Vinciguerra P, Roberts CJ, Ghaffari R, Asgari S (2019) Two-year changes in corneal stiffness parameters after accelerated corneal cross-linking. J Biomech https://doi.org/10.1016/j.jbiomech.2019.06.011

49. Elsheikh A, Alhasso D, Rama P (2008) Biomechanical properties of human and porcine corneas. Exp Eye Res 86:783-790. https:// doi.org/10.1016/j.exer.2008.02.006

50. Schultz DS, Lotz JC, Lee SM, Trinidad ML, Stewart JM (2008) Structural factors that mediate scleral stiffness. Invest Ophthalmol Vis Sci 49:4232-4236. https://doi.org/10.1167/iovs.08-1970

51. Schumacher S, Oeftiger L, Mrochen M (2011) Equivalence of biomechanical changes induced by rapid and standard corneal cross-linking, using riboflavin and ultraviolet radiation. Invest Ophthalmol Vis Sci 52: 9048-9052. https://doi.org/10.1167/iovs.11-7818

52. Krueger RR, Herekar S, Spoerl E (2014) First proposed efficacy study of high versus standard irradiance and fractionated riboflavin/ ultraviolet a cross-linking with equivalent energy exposure. Eye Contact Lens 40:353-357. https://doi.org/10.1097/ICL. 0000000000000095

Publisher's note Springer Nature remains neutral with regard to jurisdictional claims in published maps and institutional affiliations. 